\title{
Safety of Remdesivir for Patients 80 Years of Age or Older with Coronavirus Disease 2019 (COVID-19)
}

\author{
Osamu Kanai ${ }^{1,2}\left(\mathrm{~K}_{0} \cdot \mathrm{Kohei} \mathrm{Fujita}^{1,2} \cdot \mathrm{Kazutaka} \mathrm{Nanba}^{1,3} \cdot\right.$ Naoki Esaka $^{1,4} \cdot$ Hiroaki Hata $^{1,5} \cdot$ Koichi Seta $^{1,6} \cdot$ \\ Akihiro Yasoda ${ }^{7} \cdot$ Takao Odagaki $^{1} \cdot$ Tadashi Mio $^{2}$
}

Accepted: 17 November 2021 / Published online: 2 December 2021

(c) The Author(s), under exclusive licence to Springer Nature Switzerland AG 2021

\begin{abstract}
Background and Objective Although older patients with coronavirus disease 2019 (COVID-19) are at the high risk of exacerbation that requires treatment with remdesivir, the safety of this medication is unclear in clinical practice, especially among older patients. We aimed to retrospectively evaluate the safety of remdesivir in older patients with COVID-19 who required hospitalization in our institute.

Methods We reviewed patients with COVID-19 who were treated with remdesivir at our institute between July 2020 and May 2021. We defined older patients as those aged 80 years or older at admission; all other patients were defined as younger patients. We evaluated the safety of remdesivir by examining the incidence of discontinuation of remdesivir treatment because of adverse events and the incidence of any adverse events.

Results A total of 80 patients were included in this study. Compared with younger patients, fewer older patients were treated with remdesivir for more than 5 days: 4 (15.4\%) vs 23 (42.6\%). Discontinuation of remdesivir because of adverse events occurred in one older patient (3.9\%) and four younger patients (7.4\%) $[p>0.99]$. Remdesivir-induced liver dysfunction was the most frequent adverse event, which occurred in 29 (36.3\%) patients. There were no significant differences in the incidence of remdesivir-induced liver dysfunction, renal dysfunction, and fatigue.

Conclusions The safety of remdesivir was suggested to be comparable between patients older than 80 years of age and patients younger than 80 years of age. The results of this study may encourage the administration of remdesivir to this older patient group.
\end{abstract}

Osamu Kanai

okanai.kmc@gmail.com

1 Department of Infectious Diseases, National Hospital Organization Kyoto Medical Center, 1-1 Fukakusa-Mukaihata-Cho, Fushimi-Ku, Kyoto, Japan

2 Division of Respiratory Medicine, Center for Respiratory Diseases, National Hospital Organization Kyoto Medical Center, Kyoto, Japan

3 Department of Endocrinology and Metabolism, National Hospital Organization Kyoto Medical Center, Kyoto, Japan

4 Department of Gastroenterology, National Hospital Organization Kyoto Medical Center, Kyoto, Japan

5 Department of Surgery, National Hospital Organization Kyoto Medical Center, Kyoto, Japan

6 Department of Nephrology, National Hospital Organization Kyoto Medical Center, Kyoto, Japan

\section{Key Points}

Among 26 patients 80 years of age or over and 54 younger patients who were treated with remdesivir, one (3.9\%) older and four (7.4\%) younger patients discontinued the treatment because of adverse events $(p>0.99)$.

Remdesivir-induced adverse events were observed in 13 $(50.0 \%)$ older patients vs $20(37.0 \%)$ younger patients ( $p$ $=0.3346)$.

The safety of remdesivir was suggested to be comparable between older and younger patients.

7 Clinical Research Center, National Hospital Organization Kyoto Medical Center, Kyoto, Japan 


\section{Introduction}

Since severe acute respiratory syndrome coronavirus 2 (SARS-CoV-2) was first discovered in China in late 2019, coronavirus disease 2019 (COVID-19) [the disease caused by SARS-CoV-2] has since become a global pandemic [1]. As of May 2021, over 170 million people were confirmed to have a diagnosis of COVID-19 worldwide, and approximately 400,000 patients with COVID-19 have died [2,3]. In Japan, the most rapidly aging society in the world, elderly patients with COVID-19 occupy an especially high proportion of hospital beds in isolation areas [4]. Elderly patients with COVID-19 have a high risk of severe and fatal complications because older age is one of the predictive factors for poor outcome [4-6]. Improving treatments for elderly patients with COVID-19 is important as these individuals are likely to be hospitalized for longer durations and are more likely to require additional medical resources because of their impaired physical conditions and comorbidities [7].

Remdesivir, a viral RNA-dependent RNA polymerase inhibitor, is currently the only available antiviral drug that is effective against moderate-to-severe SARS-CoV-2; it was approved by the US Food and Drug Administration and the Ministry of Health, Labour and Welfare in Japan for COVID$19[8,9]$. Among adverse events commonly induced by remdesivir, the elevation of aminotransferases and/or serum creatinine requires special attention because remdesivirinduced liver and renal dysfunction may become serious. Therefore, remdesivir should be carefully administered to elderly patients because of their reduced visceral function.

Elderly patients with COVID-19 have a high risk of exacerbation that requires treatment with remdesivir, though the safety of remdesivir, especially among elderly patients, is unclear in clinical practice. At present, there is no other effective antiviral drug for treating moderate-to-severe COVID-19; therefore, knowledge of the safety of remdesivir for elderly patients is essential. We aimed to retrospectively evaluate the safety of remdesivir in elderly patients with COVID-19 who were hospitalized at our institute.

\section{Methods}

This retrospective study was conducted at the National Hospital Organization Kyoto Medical Center. We reviewed the medical records of patients with COVID-19 who were admitted to our institute. This study complied with the principles of the World Medical Association Declaration of Helsinki. This study was also reviewed and approved by the institutional review board (approval number: 21-015). We announced our intention to conduct this study and gave patients the opportunity to enroll or decline. None of the authors had conflicts of interest to declare. Cornavirus disease-2019 Registry Japan
(COVIREGI-JP) of National Center for Global Health and Medicine was used for the present study with permission. The study data were collected and managed using Research Electronic Data Capture (REDCap) a secure, web-based data capture application hosted at JCRAC data center of National Center for Global Health and Medicine.

We identified patients with COVID-19 who were treated with remdesivir at our institute between July 2020 and May 2021. All patients were diagnosed with COVID-19 by polymerase chain reaction tests for SARS-CoV-2 in nasopharyngeal swabs (SARS-CoV-2 test). Remdesivir was administered to the patients who met the following criteria: $\mathrm{SpO}_{2}$ less than $95 \%$ on room air or any radiological evidence of pneumonia, aminotransferases less than five times the upper limit of normal, estimated glomerular filtration rate (eGFR) more than 30 $\mathrm{mL} / \mathrm{min} / 1.73 \mathrm{~m}^{2}$, no allergy to remdesivir or its additives, and written informed consent from the patient or their relatives.

To eliminate the impact of other treatment agents (other than $6 \mathrm{mg} /$ day of dexamethasone or the corresponding dose of corticosteroids), we excluded patients who were treated with favipiravir, tocilizumab, baricitinib, and steroid pulse therapy [10-15]. We also excluded patients who were transferred to another hospital before completing treatment with remdesivir because we could not fully evaluate adverse events among these individuals.

All patients were treated with intravenous remdesivir at a loading dose of $200 \mathrm{mg}$ on day 1 and a maintenance dose of $100 \mathrm{mg}$ from day 2 until day 10 or until any cause of discontinuation $[8,16]$. Most patients were also treated with dexamethasone at a dose of $6 \mathrm{mg}$ once daily or the corresponding dose of corticosteroids for up to 10 days [10].

Patients were dichotomized according to their age at admission: 80 years or over (older patients) vs under 80 years (younger patients). We obtained the following clinical data from electronic medical records: age at admission, sex, coexisting conditions (hypertension, diabetes mellitus, dyslipidemia, obesity, liver dysfunction, and renal dysfunction), results of blood tests, and clinical status of the patients assessed on the National Institute of Allergy and Infectious Diseases Ordinal Scale (NIAID-OS): an eight-category ordinal scale score from 1 (not hospitalized and no limitation of activities) to 7 (hospitalized, receiving invasive mechanical ventilation or extracorporeal membrane oxygenation) and 8 (death). Because sufficient anthropometric data could not be obtained from a subset of patients, overweight and obesity were defined as $25 \mathrm{~kg} / \mathrm{m}^{2}$ or over in body mass index or as the appearance of obesity [17]. Liver dysfunction was defined as a level of total bilirubin, aspartate aminotransferase, or alanine aminotransferase above the upper normal limit. Renal dysfunction was defined as a decreased eGFR less than $60 \mathrm{~mL} / \mathrm{min} / 1.73 \mathrm{~m}^{2}$. As all participants were Japanese, the eGFR was estimated by the Japanese GFR estimating formula instead of the Modification of Diet in Renal Disease formula described below [18]. 
eGFR $\left(\mathrm{mL} / \mathrm{min} / 1.73 \mathrm{~m}^{2}\right)=194 *$ [serum creatinine $(\mathrm{mg} / \mathrm{dL})]^{\wedge}(-1.094) *[\text { age }(\text { years })]^{\wedge}(-0.287)(* 0.739$ if women)

We evaluated the safety of remdesivir by the incidence of discontinuation of remdesivir treatment due to adverse events. The causality between remdesivir and adverse events was determined clinically by physicians (O.K., K.F., H.H., and T.O.), including three Fellows of the Japanese Society of Internal Medicine, one Fellow of the Japanese Association of Infectious Diseases, two Board Certified Members of the Japanese Association of Infectious Diseases, and two Senior Fellows of the Japanese Respiratory Society. The discrepancy of the judgment was solved by discussion. We evaluated the severity of any adverse events in accordance with the Common Terminology Criteria for Adverse Events version 5.0 [19].

We assessed the outcome of patients with COVID-19 who were treated with remdesivir by the following criteria: mortality within 60 days of admission and improvement in the NIAID-OS at discharge from the isolation area of our institute. The characteristics of the patients were described according to the age at admission (older vs younger patients). Categorical variables and ordinal variables (NIAID-OS and a grade of remdesivir-induced adverse events calculated with Common Terminology Criteria for Adverse Events version 5.0) are presented as numbers and percentages, and continuous variables (age and the results of blood tests) are presented as medians and ranges. Categorical variables were compared with Fisher's exact tests, and continuous variables and ordinal variables were compared using Mann-Whitney $U$ tests. Nonparametric tests were used for not only ordinal variables but also continuous variables because of the existence of outliers and the uncertainty of homoscedasticity. Statistical tests were two-sided, and the alpha value was set to 0.05 . All statistical analyses were performed by JMP version 15.2.0 software (SAS Institute, Inc., Cary, NC, USA).

\section{Results}

Between July 2020 and May 2021, 150 patients with COVID-19 were admitted to the National Hospital Organization Kyoto Medical Center. Among 108 patients who were treated with remdesivir at our institute, 80 patients met the inclusion criteria for the present study (Fig. 1). Twenty-six $(32.5 \%)$ patients were aged 80 years or over (older patients), and 54 (67.5\%) patients were aged under 80 years (younger patients). The baseline characteristics of the patients according to age are described in Table 1 . Women were predominant among older patients, whereas men were predominant among younger patients; the difference in sex profile between the groups was not statistically significant. The prevalence of overweight and obesity

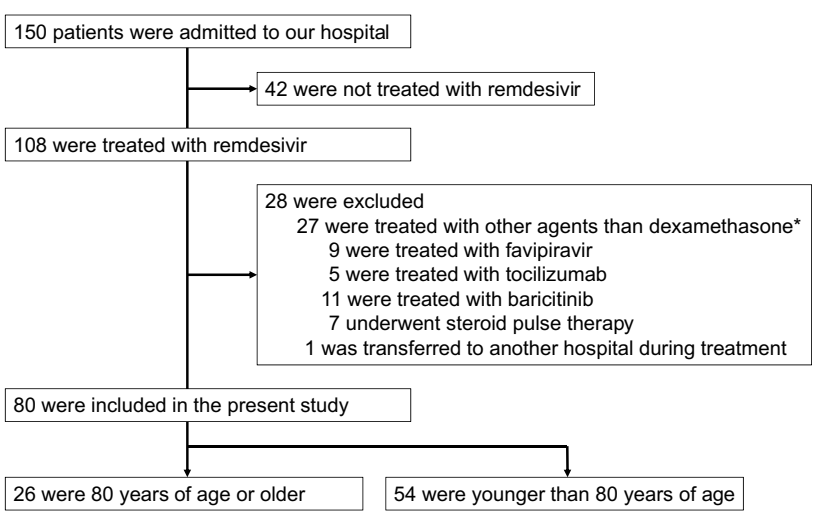

Fig. 1 Study flowchart. Screening, patient selection, exclusion, and classification. *There is duplication in concomitant agents other than dexamethasone

among older patients was significantly less than that among younger patients (3 [11.5\%] vs 19 [35.2\%], $p=0.0330)$. The prevalence of older patients with a score of 7 on the NIAIDOS was lower than that of younger patients (2 [7.7\%] vs 12 [22.2\%]); however, there was no significant difference in the severity scores of the NIAID-OS at admission between the groups ( $p=0.1956)$. In blood tests performed at admission, serum aspartate aminotransferase $(p=0.282)$, alanine aminotransferase ( $p=0.0007)$, eGFR $(p=0.0015)$, and C-reactive protein $(p=0.0414)$ were significantly lower in older patients. Although there was no significant difference in the prevalence of patients with liver dysfunction at admission (12 [46.2\%] vs 35 [64.8\%], $p=0.1472$ ), the prevalence of renal dysfunction in older patients was significantly higher than that in younger patients (20 [76.9\%] vs 19 [35.2\%], $p$ $=0.0007$ ).

Remdesivir was administered for a median of 5 [range $2-10$ ] days in both older and younger patients, though the difference was statistically significant $(p=0.0453)$ (Table 2). This significant difference occurred because compared with younger patients, fewer older patients were treated with remdesivir for more than 5 days: 4 (15.4\%) vs $23(42.6 \%)$ in older and younger patients, respectively $(p=$ 0.0224). Almost all patients received dexamethasone concurrently, and a similar proportion of patients in each group used anticoagulants: 9 (34.6\%) vs $21(38.9 \%)$ in older and younger patients, respectively $(p=0.8076)$.

There was no significant difference in the proportion of patients who discontinued remdesivir because of adverse events: one (3.9\%) in older patients and four $(7.4 \%)$ in younger patients $(p>0.99)$. Among patients who discontinued remdesivir, one older patient was administered remdesivir for 2 days, and four younger patients were administered remdesivir for 2, 3, 4, and 5 days, separately. Four out of 5 patients discontinued remdesivir because of severe liver dysfunction, and the remaining patient discontinued remdesivir 
Table 1 Baseline characteristics of patients with coronavirus disease 2019 (COVID-19) who were treated with remdesivir

\begin{tabular}{|c|c|c|c|c|}
\hline & Total $(n=80)$ & Older patients $(n=26)$ & Younger patients $(n=54)$ & $P$ value \\
\hline Age (years) & 73 [39-95] & $87[80-95]$ & 68 [39-79] & - \\
\hline Sex (male) & $47(58.8)$ & $11(42.3)$ & $36(66.7)$ & 0.0528 \\
\hline \multicolumn{5}{|l|}{ Coexisting condition } \\
\hline Hypertension & $36(45.0)$ & $12(46.2)$ & $24(44.4)$ & $>0.99$ \\
\hline Diabetes mellitus & $24(30.0)$ & $9(34.6)$ & $15(27.8)$ & 0.6057 \\
\hline Dyslipidemia & $20(25.0)$ & $5(19.2)$ & $15(27.8)$ & 0.5825 \\
\hline Obesity & $22(27.5)$ & $3(11.5)$ & $19(35.2)$ & 0.0330 \\
\hline \multicolumn{5}{|l|}{ NIAID-OS at admission } \\
\hline $\begin{array}{l}\text { 4. Hospitalized, not requiring supplemental oxygen, requir- } \\
\text { ing ongoing medical care }\end{array}$ & $22(27.5)$ & $8(30.8)$ & $14(25.9)$ & $0.1956^{\mathrm{a}}$ \\
\hline 5. Hospitalized, requiring supplemental oxygen & $25(31.3)$ & $10(38.5)$ & $15(27.8)$ & \\
\hline $\begin{array}{l}\text { 6. Hospitalized, receiving noninvasive ventilation or high- } \\
\text { flow oxygen devices }\end{array}$ & $19(23.8)$ & $6(23.1)$ & $13(24.1)$ & \\
\hline $\begin{array}{l}\text { 7. Hospitalized, receiving invasive mechanical ventilation or } \\
\text { extracorporeal membrane oxygenation }\end{array}$ & $14(17.5)$ & $2(7.7)$ & $12(22.2)$ & \\
\hline \multicolumn{5}{|l|}{ Blood sample tests on admission } \\
\hline \multicolumn{5}{|l|}{ Blood cell counts } \\
\hline $\mathrm{WBC}\left(\times 10^{\wedge} 9 / \mathrm{L}\right)$ & $5.35[0.7-13.6]$ & $5.15[0.7-12.4]$ & $5.35[1.9-13.6]$ & 0.7346 \\
\hline Hemoglobin (g/L) & 132 [49-170] & 122 [89-169] & $136.5[49-170]$ & 0.0666 \\
\hline Platelet $\left(\times 10^{\wedge} 9 / \mathrm{L}\right)$ & $170[14.9-425]$ & 154 [39-312] & $176.5[14.9-425]$ & 0.1391 \\
\hline \multicolumn{5}{|l|}{ Biochemical tests } \\
\hline Total protein $(\mathrm{g} / \mathrm{L})$ & $67[47-89]$ & $66.5[53-89]$ & $67[47-76]$ & 0.4832 \\
\hline Albumin (g/L) & 33 [19-45] & $31[25-39]$ & $33.5[19-45]$ & 0.2125 \\
\hline Total bilirubin (mg/dL) & $0.55[0.2-2.3]$ & $0.5[0.2-2.3]$ & $0.6[0.3-1.3]$ & 0.8977 \\
\hline AST (IU/L) & $37[15-285]$ & $29.5[15-88]$ & 37 [15-285] & 0.0282 \\
\hline ALT (IU/L) & $25[5-225]$ & $17[5-104]$ & $28.5[7-225]$ & 0.0007 \\
\hline Lactate dehydrogenase (IU/L) & $304.5[142-851]$ & $284.5[142-656]$ & $307.5[178-851]$ & 0.2294 \\
\hline Creatine kinase (U/L) & 96.5 [15-2119] & $91.5[15-1252]$ & 117 [26-2119] & 0.2947 \\
\hline Creatinine (mg/dL) & $0.855[0.2-1.82]$ & $0.88[0.2-1.72]$ & $0.83[0.37-1.82]$ & 0.3444 \\
\hline $\mathrm{eGFR}\left(\mathrm{mL} / \mathrm{min} / 1.73 \mathrm{~m}^{2}\right)$ & $61.3[30.5-228.5]$ & $50.55[30.5-228.5]$ & $66[32.6-124.2]$ & 0.0015 \\
\hline Glucose (mg/dL) & $126[64-285]$ & $118.5[64-285]$ & 128 [78-283] & 0.1056 \\
\hline C-reactive protein $(\mathrm{mg} / \mathrm{L})$ & $73.65[4.7-306.4]$ & $54.65[5.6-206.4]$ & $79.35[4.7-306.4]$ & 0.0414 \\
\hline \multicolumn{5}{|l|}{ Coagulation system } \\
\hline PT-INR & $0.975[0.85-4.01]$ & $0.965[0.85-1.36]$ & $0.98[0.85-4.01]$ & 0.4622 \\
\hline APTT (sec) & $30.45[10-120]$ & $29[22.1-120]$ & $31[10-84.8]$ & 0.5478 \\
\hline Liver dysfunction on admission & $47(58.8)$ & $12(46.2)$ & $35(64.8)$ & 0.1472 \\
\hline Renal dysfunction on admission & $39(48.8)$ & $20(76.9)$ & $19(35.2)$ & 0.0007 \\
\hline
\end{tabular}

Data are shown with number and (percentage) or median and [range]. Obesity is defined as $25 \mathrm{~kg} / \mathrm{m}^{2}$ or more in body mass index or as the appearance of obesity. Liver dysfunction is defined as a level of total bilirubin, AST, or ALT above the upper normal limit. Renal dysfunction is defined as a level of eGFR less than $60 \mathrm{~mL} / \mathrm{min} / 1.73 \mathrm{~m}^{2}$

$A L T$ alanine aminotransferase, APTT activated partial thromboplastin time, $A S T$ aspartate aminotransferase, $e G F R$ estimated glomerular filtration rate, NIAID-OS National Institute of Allergy and Infectious Diseases Ordinal Scale, PT-INR prothrombin time-international normalized ratio, $W B C$ white blood cell, sec seconds

${ }^{a}$ Mann-Whitney $U$ test was used for comparing the NIAID-OS

because of grade 2 renal dysfunction. Remdesivir-induced adverse events were observed in $13(50.0 \%)$ older patients vs $20(37.0 \%)$ younger patients $(p=0.3346)$. Among all adverse events induced by remdesivir, liver dysfunction was the most frequent event, occurring in 29 (36.3\%) patients.
There were no significant differences between older and younger patients in the grade of remdesivir-induced liver dysfunction ( $p=0.4294)$ and severe liver dysfunction (one [3.9\%] vs three [5.6\%] in older and younger patients, respectively $[p>0.99])$. Similar to remdesivir-induced liver 
Table 2 Treatment with remdesivir and concomitant agents, and remdesivir-induced adverse events

\begin{tabular}{|c|c|c|c|c|}
\hline & $\begin{array}{l}\text { Total } \\
(n=80)\end{array}$ & $\begin{array}{l}\text { Older patients } \\
(n=26)\end{array}$ & $\begin{array}{l}\text { Younger patients } \\
(n=54)\end{array}$ & $P$ value \\
\hline Treatment duration of remdesivir (days) & $5[2-10]$ & $5[2-10]$ & $5[2-10]$ & 0.0453 \\
\hline Treatment with remdesivir for more than 5 days & $27(33.8)$ & $4(15.4)$ & $23(42.6)$ & 0.0224 \\
\hline Concomitant use of corticosteroids & $79(98.8)$ & $25(96.2)$ & $54(100.0)$ & 0.325 \\
\hline Concomitant use of anticoagulants & $30(37.5)$ & $9(34.6)$ & $21(38.9)$ & 0.8076 \\
\hline Discontinuation of remdesivir & $5(6.3)$ & $1(3.9)$ & $4(7.4)$ & $>0.99$ \\
\hline Any adverse events induced by remdesivir & $33(41.3)$ & $13(50.0)$ & $20(37.0)$ & 0.3346 \\
\hline \multicolumn{5}{|l|}{ Remdesivir-induced liver dysfunction } \\
\hline Grade: 0 & $51(63.8)$ & $14(53.9)$ & $37(68.5)$ & \multirow[t]{4}{*}{$0.4294^{\mathrm{a}}$} \\
\hline 1 & $20(25.0)$ & $11(42.3)$ & $9(16.7)$ & \\
\hline 2 & $5(6.3)$ & $0(0.0)$ & $5(9.3)$ & \\
\hline 3 & $4(5.0)$ & $1(3.9)$ & $3(5.6)$ & \\
\hline Any grade of liver dysfunction & $29(36.3)$ & $12(46.2)$ & $17(31.5)$ & 0.2230 \\
\hline Severe liver dysfunction & $4(5.0)$ & $1(3.9)$ & $3(5.6)$ & $>0.99$ \\
\hline \multicolumn{5}{|l|}{ Remdesivir-induced renal dysfunction } \\
\hline Grade: 0 & $76(95.0)$ & $25(96.2)$ & $51(94.4)$ & \multirow[t]{3}{*}{$0.7441^{\mathrm{a}}$} \\
\hline 1 & $3(3.8)$ & $1(3.9)$ & $2(3.7)$ & \\
\hline 2 & $1(1.3)$ & $0(0.0)$ & $1(1.9)$ & \\
\hline Any grade of renal dysfunction & $4(5.0)$ & $1(3.9)$ & $3(5.6)$ & $>0.99$ \\
\hline \multicolumn{5}{|l|}{ Remdesivir-induced fatigue } \\
\hline Grade: 0 & $79(99.0)$ & $26(100)$ & $53(98.1)$ & \multirow[t]{2}{*}{$0.5046^{\mathrm{a}}$} \\
\hline 2 & $1(1.3)$ & $0(0.0)$ & $1(1.9)$ & \\
\hline
\end{tabular}

Data are shown with number and (percentage) or median and [range]. The severity of adverse events is evaluated in accordance with the Common Terminology Criteria for Adverse Events version 5.0. Severe liver dysfunction is defined as grade 3 or more severe liver dysfunction

${ }^{a}$ Mann-Whitney $U$ tests were used for comparing the grade of severity

dysfunction, there was no significant difference in the grade $(p=0.2230)$ and occurrence of remdesivir-induced renal dysfunction (one [3.9\%] vs three [5.6\%] in older and younger patients, respectively [ $p>0.99])$. Remdesivir-induced fatigue was observed in only one younger patient and not in the older patient. We could find remdesivir-induced fatigue by the following course. The patient required $1 \mathrm{~L} / \mathrm{min}$ of oxygen supply for maintaining above $95 \%$ of $\mathrm{SpO}_{2}$ but had no other symptom. However, the patient developed appetite loss and fatigue without fever and progression of respiratory symptoms after the initial administration of remdesivir. After 5 days of treatment with remdesivir, the patient rapidly recovered from the fatigue on the sixth day. Other than these events, there were no previously reported adverse events that would be of particular concern to elderly patients.

The duration from admission to discharge from an isolated unit for COVID-19 was 12.5 [7-35] days vs 13 [8-59] days in older and younger patients, respectively ( $p=0.7559)$ (Table 3). At discharge from the isolation area, there was no significant difference in NIAID-OS between the groups ( $p$ $=0.0758$ ). During the period, improvement in NIAID-OS was observed in $15(57.7 \%)$ older patients and 44 (81.5\%) younger patients $(p=0.0314)$, whereas six $(23.1 \%)$ older patients and seven $(13.0 \%)$ younger patients died from COVID-19 $(p=0.3331)$ within 60 days of admission.

\section{Discussion}

In this retrospective study, we found no statistically significant differences between older and younger patients in the proportion of patients who discontinued remdesivir because of adverse events, such as liver dysfunction, renal dysfunction, and fatigue. Regarding the clinical outcome of COVID-19, younger patients achieved clinical improvement at discharge from isolated areas more frequently than older patients. The mortality rate within 60 days of a COVID19 diagnosis was higher in older patients than in younger patients, but the difference was not significant.

In the whole cohort, the incidence of discontinuation of remdesivir was $6.3 \%$, which was slightly lower than that in the Adaptive COVID-19 Treatment Trial (ACTT)-1 study (9.8\%) [8]. Considering the higher mean age of this study cohort compared with that of the ACTT-1 study (71.4 vs 58.6 years old), older age does not appear to be associated 
Table 3 Treatment outcome of patients with coronavirus disease 2019 (COVID-19)

\begin{tabular}{|c|c|c|c|c|}
\hline & $\begin{array}{l}\text { Total } \\
(n=80)\end{array}$ & $\begin{array}{l}\text { Older patients } \\
(n=26)\end{array}$ & $\begin{array}{l}\text { Younger } \\
\text { patients } \\
(n=54)\end{array}$ & $P$ value \\
\hline Duration of admission $^{\mathrm{a}}$ (days) & $13[7-59]$ & $12.5[7-35]$ & 13 [8-59] & 0.7559 \\
\hline \multicolumn{5}{|l|}{ NIAID-OS at discharge from isolation area } \\
\hline 1. Not hospitalized, no limitations of activities & $32(40.0)$ & $5(19.2)$ & $27(50.0)$ & $0.0758^{\mathrm{b}}$ \\
\hline $\begin{array}{l}\text { 2. Not hospitalized, limitation of activities, home oxygen requirement, or } \\
\text { both }\end{array}$ & $1(1.3)$ & $0(0.0)$ & $1(1.9)$ & \\
\hline $\begin{array}{l}\text { 3. Hospitalized, not requiring supplemental oxygen and no longer requiring } \\
\text { ongoing medical care }\end{array}$ & $7(8.8)$ & $4(15.4)$ & $3(5.6)$ & \\
\hline $\begin{array}{l}\text { 4. Hospitalized, not requiring supplemental oxygen, requiring ongoing medi- } \\
\text { cal care }\end{array}$ & $11(13.8)$ & $8(30.8)$ & $3(5.6)$ & \\
\hline 5. Hospitalized, requiring supplemental oxygen & $15(18.8)$ & $3(11.5)$ & $12(22.2)$ & \\
\hline $\begin{array}{l}\text { 6. Hospitalized, receiving noninvasive ventilation or high-flow oxygen } \\
\text { devices }\end{array}$ & $0(0.0)$ & $0(0.0)$ & $1(1.9)$ & \\
\hline $\begin{array}{l}\text { 7. Hospitalized, receiving invasive mechanical ventilation or extracorporeal } \\
\text { membrane oxygenation }\end{array}$ & $1(1.3)$ & $0(0.0)$ & $1(1.9)$ & \\
\hline 8. Died & $13(16.3)$ & $6(23.1)$ & $7(13.0)$ & \\
\hline Improvement in NIAID-OS & $59(67.0)$ & $15(57.7)$ & $44(81.5)$ & 0.0314 \\
\hline Death within 60 days of admission & $13(16.3)$ & $6(23.1)$ & $7(13.0)$ & 0.3331 \\
\hline
\end{tabular}

Data are shown with number and (percentage) or median and [range]

NIAID-OS National Institute of Allergy and Infectious Diseases Ordinal Scale

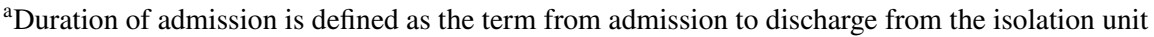

${ }^{\mathrm{b}}$ Mann-Whitney $U$ tests were used for comparing the NIAID-OS

with a higher incidence of discontinuation of remdesivir due to adverse events despite different patient backgrounds.

Based on the incidence of any adverse events and discontinuation of remdesivir because of adverse events in the present study, remdesivir is unlikely to be a drug with special safety concerns for elderly patients. Although older patients were administered remdesivir for a shorter time than younger patients, all discontinuations of remdesivir occurred within 5 days; a shorter treatment duration in older patients did not seem to affect the incidence of treatment discontinuation. The main reason for the discontinuation of remdesivir was severe liver dysfunction, which was the most frequently observed adverse event. Despite the significantly higher prevalence of renal dysfunction in older patients on admission, remdesivir-induced renal dysfunction did not adversely affect the discontinuation of remdesivir. In contrast, most patients with renal dysfunction at admission improved after treatment for COVID-19 (data are not shown). This may be the result of dehydration due to fever and loss of appetite, which are especially likely to occur in elderly patients.

Older patients were less likely to achieve improvement in severity scores than younger patients [5]. Moreover, the mortality rate of COVID-19 within 60 days of diagnosis was higher in older patients, as expected [4, 6]. As we previously reported, older patients in this study usually lived at a nursing care home or had complications requiring hospitalization, such as bedridden status, feeding difficulty, disquiet, delirium, dementia, and continuous sputum suction needs [7]. These patients required ongoing hospitalization after release from isolation. Ultimately, these patients may not achieve an improvement in NIAID-OS scores even if they recover from COVID-19. Among elderly patients, a significantly shorter treatment duration may not the main reason for a lack of improvement in clinical status. This is because treatment durations of 5 days or 10 days for patients who did not need mechanical ventilation did not lead to a significant difference in improvement of clinical status $[9,16]$. No significant improvement in mortality rate could be observed because of the insufficient number of patients in this study.

This study has some limitations. First, this study included an insufficient number of patients to perform a multivariate analysis for the association between older age and the safety of remdesivir. Second, the baseline characteristics of each group were not well balanced, including the prevalence of overweight and obesity, NIAID-OS at admission, and results of blood tests. The older patients may have a NIAID-OS of 5 or less, despite their critically ill condition. This is because they may refuse invasive treatment such as 
high-flow oxygen therapy and mechanical ventilation, which correspond to 6 or 7 on the NIAID-OS. Third, because of the retrospective nature of this study, we could not quantitatively evaluate several factors that may affect the prognosis of COVID-19, including cardiac function, respiratory function, and activities of daily living at admission. Fourth, generalizability of the results was not fully warranted because this study was conducted at a single institute and consisted of Asian patients only. Finally, the effects of corticosteroid therapy could not be excluded because almost all patients were treated with dexamethasone or other corticosteroids. Although there are several limitations, it was a remarkable result that almost all older patients completed the treatment with remdesivir despite having complications. The results of this study may encourage, rather than raise special concern for administering remdesivir to elderly patients.

\section{Conclusions}

The safety of remdesivir was suggested to be comparable between patients older than 80 years of age and patients younger than 80 years of age, as evaluated by the incidence of treatment discontinuation and any adverse events. As the discontinuation of treatment with remdesivir occurs during days 2 through 5 , we should pay particular attention to the occurrence of adverse events in the early stages of treatment. Further study for investigating the safety of remdesivir for elderly patients is warranted.

Acknowledgements We thank American Journal Experts (http://www. aje.com) for their help with English language editing. COVIREGI-JP of National Center for Global Health and Medicine were used for this study with permission.

\section{Declarations}

Funding This study was supported in part by a grant from the National Hospital Organization's fiduciary funds (for English editing).

\section{Conflicts of interest/competing interests Not applicable.}

Ethics approval This study has been reviewed and approved by an institutional review board (approval number: 21-015).

Consent to participate We have announced our intention to conduct this study and have given patients (or their relatives) the opportunity to enroll or decline.

Consent for publication We have announced our intention to conduct this study and to publish the results of the study, and we have given patients (or their relatives) the opportunity to enroll or decline.

Availability of data and material Because of the method for informed consent of this study, we could not confirm the agreement for their data to be shared publicly, thus supporting data are not available.
Code availability Not applicable.

Author contributions Conceptualization, OK, TO, and TM; methodology, OK, KF, and TM; software, OK; validation, OK and TM; formal analysis, $\mathrm{OK}$; data curation, $\mathrm{OK}, \mathrm{KF}, \mathrm{HH}$, and $\mathrm{TO}$; writing (original draft preparation), OK; writing (review and editing), KF, KN, NE, KS, $\mathrm{AY}$, and TM; visualization, OK; supervision, $\mathrm{HH}, \mathrm{KS}, \mathrm{AY}$, TO, and TM; project administration, AY, TO, and TM. All authors have read and agreed to the published version of the manuscript.

\section{References}

1. Zhu N, Zhang D, Wang W, Li X, Yang B, Song J, et al. A novel coronavirus from patients with pneumonia in China, 2019. N Engl J Med. 2020;382:727-33.

2. World Health Organization. Coronavirus (COVID-19) cases. https://ourworldindata.org/covid-cases. Accessed 14 Jul 2021.

3. World Health Organization. Coronavirus (COVID-19) deaths. https://ourworldindata.org/covid-deaths. Accessed 14 Jul 2021.

4. Ministry of Health, Labour and Welfare. Open data for COVID19 in Japan. https://www.mhlw.go.jp/stf/covid-19/open-data.html. Accessed 14 Jul 2021.

5. Gao YD, Ding M, Dong X, Zhang JJ, Kursat Azkur A, et al. Risk factors for severe and critically ill COVID-19 patients: a review. Allergy. 2021;76:428-55.

6. Onder G, Rezza G, Brusaferro S. Case-fatality rate and characteristics of patients dying in relation to COVID-19 in Italy. JAMA. 2020;323:1775-6.

7. Fujita K, Kashihara E, Kanai O, Hata H, Yasoda A, Odagaki $\mathrm{T}$, et al. Increasing burden of nursing care on the treatment of COVID-19 patients in the ageing society: analyses during the first to the third wave of pandemic in Kyoto City, Japan. Res Sq. 2021. https://doi.org/10.21203/rs.3.rs-492626/v1.

8. Beigel JH, Tomashek KM, Dodd LE, Mehta AK, Zingman BS, Kalil AC, et al. Remdesivir for the treatment of Covid-19: final report. N Engl J Med. 2020;383:1813-26.

9. National Institutes of Health. COVID-19 Treatment Guidelines Panel. Coronavirus disease 2019 (COVID-19) treatment guidelines. https://www.covid19treatmentguidelines.nih.gov/. Accessed 8 Jul 2021.

10. The RECOVERY Collaborative Group. Dexamethasone in hospitalized patients with Covid-19. N Engl J Med. 2021;384:693-704.

11. Ramanathan K, Antognini D, Combes A, Paden M, Zakhary B, Ogino $\mathrm{M}$, et al. Tocilizumab in patients admitted to hospital with COVID-19 (RECOVERY): a randomised, controlled, open-label, platform trial. Lancet. 2020;397:1637-45.

12. The REMAP-CAP Investigators. Interleukin-6 receptor antagonists in critically ill patients with Covid-19. N Engl J Med. 2021;384:1491-502.

13. Kalil AC, Patterson TF, Mehta AK, Tomashek KM, Wolfe CR, Ghazaryan V, et al. Baricitinib plus remdesivir for hospitalized adults with Covid-19. N Engl J Med. 2021;384:795-807.

14. Marconi VC, Ramanan AV, de Bono S, Kartman CE, Krishnan $\mathrm{V}$, Liao R, et al. Efficacy and safety of baricitinib in patients with COVID-19 infection: results from the randomised, double-blind, placebo-controlled, parallel-group COV-BARRIER phase 3 trial. medRxiv. 2021. https://doi.org/10.1101/2021.04.30.21255934.

15. Edalatifard M, Akhtari M, Salehi M, Naderi Z, Jamshidi A, Mostafaei $S$, et al. Intravenous methylprednisolone pulse as a treatment for hospitalised severe COVID-19 patients: results from a randomised controlled clinical trial. Eur Respir J. 2020;56:2002808. https://doi.org/10.1183/13993003.02808-2020. 
16. Goldman JD, Lye DCB, Hui DS, Marks KM, Bruno R, Montejano $\mathrm{R}$, et al. Remdesivir for 5 or 10 days in patients with severe Covid19. N Engl J Med. 2020;383:1827-37.

17. Lim JU, Lee JH, Kim JS, Hwang YI, Kim TH, Lim SY, et al. Comparison of World Health Organization and Asia-Pacific body mass index classifications in COPD patients. Int J COPD. 2017;12:2465-75.

18. Matsuo S, Imai E, Horio M, Yasuda Y, Tomita K, Nitta K, et al. Revised equations for estimated GFR from serum creatinine in
Japan. Am J Kidney Dis. 2009;53:982-92. https://doi.org/10. 1053/j.ajkd.2008.12.034.

19. U.S. Department of Health and Human Services. National Institutes of Health. National Cancer Institute. Common Terminology Criteria for Adverse Events (CTCAE) version 5.0. 2017. https:// ctep.cancer.gov/protocoldevelopment/electronic_applications/ docs/ctcae_v5_quick_reference_8.5x11.pdf. Accessed 14 Jul 2021. 